\title{
A THREE-DIMENSIONAL PARALLEL TIME-ACCURATE TURBOPUMP SIMULATION PROCEDURE USING OVERSET GRID SYSTEMS
}

\author{
Cetin Kiris, kiris@nas.nasa.gov \\ William Chan, wchan@nas.nasa.gov \\ Dochan Kwak, dkwak@mail.arc.nasa.gov
}

NAS Applications Branch, M.S, T27B-1

NASA-Ames Research Center, Moffett Field, CA 94035

\begin{abstract}
The objective of the current effort is to provide a computational framework for design and analysis of the entire fuel supply system of a liquid rocket engine, including high-fidelity unsteady turbopump flow analysis. This capability is needed to support the design of pump sub-systems for advanced space transportation vehicles that are likely to involve liquid propulsion systems. To date, computational tools for design/analysis of turbopump flows are based on relatively lower fidelity methods. An unsteady, three-dimensional viscous flow analysis tool involving stationary and rotational components for the entire turbopump assembly has not been available for real-world engineering applications. The present effort provides developers with information such as transient flow phenomena at start up, and non-uniform inflows, and will eventually impact on system vibration and structures. In the proposed paper, the progress toward the capability of complete simulation of the turbo-pump for a liquid rocket engine is reported. The Space Shuttle Main Engine (SSME) turbo-pump is used as a test case for evaluation of the hybrid MPI/Open-MP and MLP versions of the INS3D code. CAD to solution auto-scripting capability is being developed for tubopump applications. The relative motion of the grid systems for the rotor-stator interaction was obtained using overset grid techniques. Unsteady computations for the SSME turbo-pump, which contains 114 zones with 34.5 million grid points, are carried out on Origin 3000 systems at NASA Ames Research Center. Results from these time-accurate simulations with moving boundary capability will be presented along with the performance of parallel versions of the code.
\end{abstract}

Until recently, the high performance pump design process was not significantly different from that of 30 years ago. During the past 30 years a vast amount of experimental and operational experience has demonstrated that there are many important features of pump flows, which are not accounted for in the semi-empirical design process. Pumps being designed today are no more technologically advanced than those designed for the Space Shuttle Main Engine (SSME). During that same time span huge strides have been made in computers, numerical algorithms, and physical modeling.

Rocket pumps involve full and partial blades, tip leakage, and exit boundary to diffuser. In addition to the geometric complexities, a variety of flow phenomena are encountered in turbopump flows. These include turbulent boundary layer

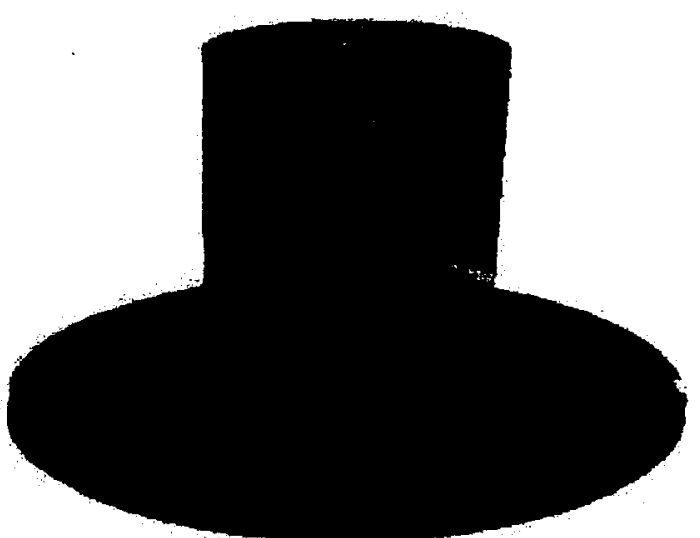

Figure 1. Geometry of a SSME pump including inlet guide vanes, impeller and diffuser blades. 
separation, wakes, transition, tip vortex resolution, threedimensional effects, and Reynolds numbers effects. In order to increase the role of Computational Fluid Dynamics (CFD) in the design process, the CFD analysis tools must be evaluated and validated so that designers gain confidence in their use. As a part of extending the CFD technology to pump design work, INS3D code has been validated for pump component analysis using data from a rocket pump inducer.

When rotating and stationary parts are included as shown in Figure 1, unsteady interactions between stationary and rotating components need to be included. To resolve the complex geometry in relative motion, an overset grid approach is employed in the current effort. In this approach, a geometrically complex body is decomposed nto a number of simple grid components. In order to solve the entire configuration including inlet guide vanes, impeller blades, and diffuser blades, an overset grid system was constructed using 34.3 Million grid points with 114 zones in the present study. Since neighboring grids may overlap arbitrarily, these grids can be created independently from each other. Thus it is easier to generate high quality and nearly orthogonal grids using this approach. Connectivity between neighboring grids is established by interpolation at the grid outer boundaries. Addition of new components to the system and simulation of arbitrary relative motion between multiple bodies are achieved by establishing new connectivity without disturbing the existing grids.

Parallel computing strategies vary depending on computer architecture such as memory arrangement relative to processing units. Two approaches have been implemented in the present study: the first approach is hybrid MPI/OpenMP and the second one is Multi Level Parallelism (MLP) developed at NASA-Ames Research Center. The first approach is obtained by using massage-passing interface (MPI) for inter-zone parallelism, and by using OpenMP directives for intra-zone parallelism. INS3D-MPI is based on the explicit messagepassing interface across MPI groups and is designed for coarse grain parallelism. The second approach is obtained by using NAS-MLP routines. This approach differs from the MPI/OpenMP approach in a fundamental way in that it does not use messaging at all. All data communication at the coarsest and finest level is accomplished via direct memory referencing instructions. This approach also provides a simpler mechanism than MPI for converting legacy code, such as INS3D. For shared memory MLP, the coarsest level parallelism is supplied by spawning of independent processes via the standard UNIX fork. The advantage of the UNIX fork over MPI procedure is that the user does not have to change the initialization section of the large production code. A library of routines is used to initiate forks, to establish shared memory arenas, and to provide synchronization primitives. The boundary data for the overset grid system is archived in the shared memory arena by each process. Other processes access the data from the arena as needed. Figure 2 shows the scalability for SSME impeller computations using 19.2 Million grid points.

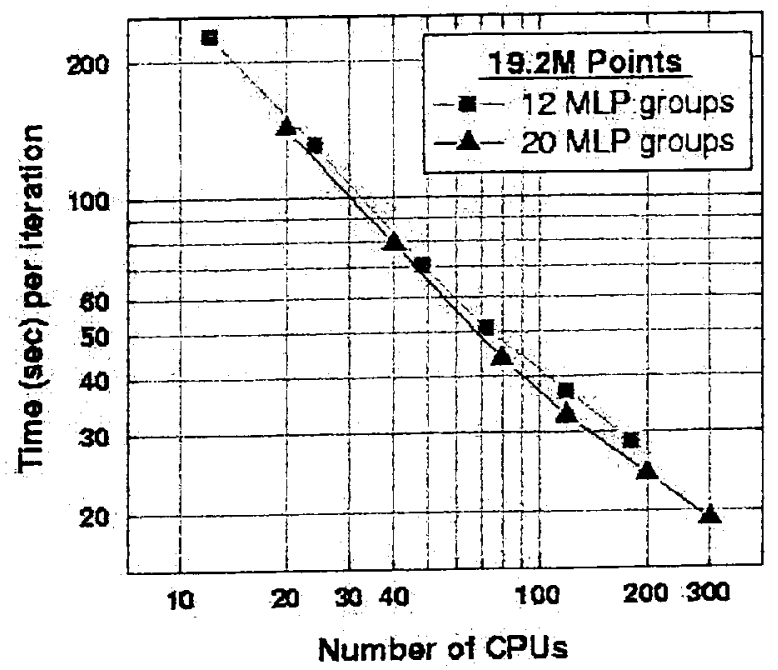

Figure 2. Time (sec) per iteration for SSME impeller computations.

Using both MPI/OpenMP hybrid approach and NAS-MLP parallel implementation, time-accurate computations of a pump shown in Figure 1 have been carried out on SGI Origin 2000 and 3000 platforms. Initially the flow was at rest and the impeller started to rotate impulsively. An instantaneous snapshot of particle traces and pressure surfaces from these computations are shown in Figure 3 after three full impeller rotations were completed. This procedure provides flow-field details not readily available from experiments. A full-length version of this work will be presented in the paper.

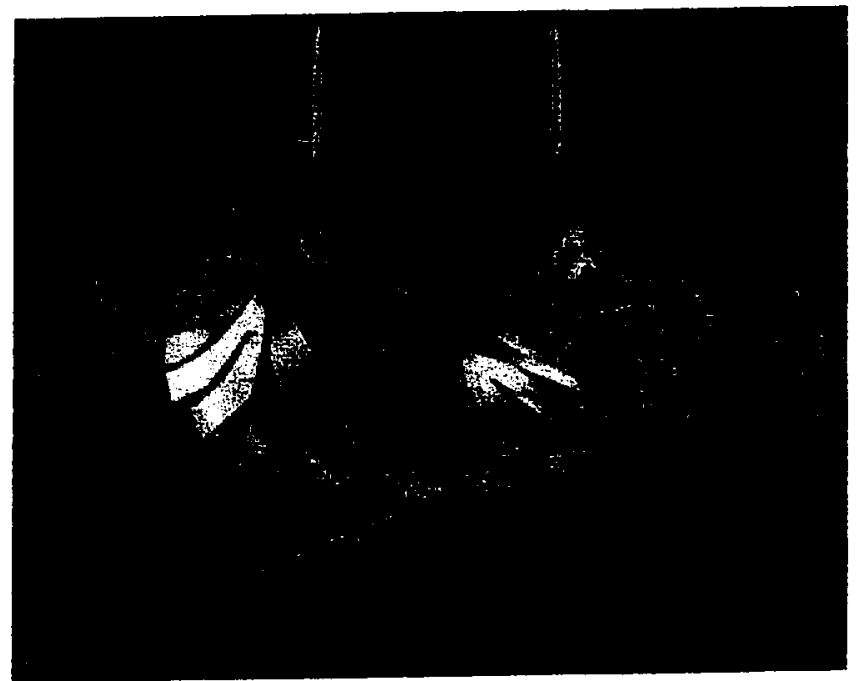

Figure 3. A snapshot of particle traces and pressure surfaces from unsteady turbopump computations. 